\title{
ON THE REFLECTION LAWS OF SECOND ORDER DIFFERENTIAL EQUATIONS IN TWO INDEPENDENT VARIABLES
}

\section{HANS LEWY}

The main purpose of this paper is the study of the analytic continuation of solutions of elliptic differential equations in two independent variables across an analytic boundary on which they satisfy further analytic equations connecting the point of the boundary, the value of the solution and those of its first order partial derivatives.

In its simplest form the problem is that of the continuation of a harmonic function of two variables $x, y$ across a segment of the $x$ axis if on this segment an analytic relation holds between $x$, the given harmonic function $u$ and its conjugate harmonic $v$, and the derivatives $u_{x}$ and $u_{y}$. This case was the subject of [2] and of [7]. Here we shall complement the methods and results of [7] by discussing the special case of a linear boundary relation. We shall see that $u$ can be extended as harmonic function into the mirror image of its domain $D$ of definition provided $D$ is simply connected. In other words: the general linear boundary condition and the boundary condition $u=0$ do not differ with respect to the domain into which they permit the class of harmonic functions on $D$ to be extended, they are "coextensive."

In this respect there is a fundamental difference between harmonic functions of two and of three variables. We shall furnish an example of a harmonic function of three variables which satisfies a linear boundary condition, even of constant coefficients, on a boundary plane, but which is not coextensive with the class of harmonic functions of the same domain of definition, but satisfying the condition $u=0$ on the boundary plane. The example is such as to permit the choice of the boundary condition in a manner to exclude an arbitrary point of the mirror image domain from the domain of analytic extension.

This example illustrates, by exhibiting the breakdown of analogy of facts, the necessity of employing in the treatment of equations in two independent variables tools especially adapted to this case. We shall see that in essence the problem of analytic continuation of

An address delivered before the Annual Meeting of the Society in Berkeley on December 30, 1954 by invitation of the Committee to Select Hour Speakers for Far Western Sectional Meetings; received by the editors July 17, 1958. 
solutions of elliptic equations in two variables can be thrown back on the problem of analytic extension of holomorphic functions of one complex variable. But the boundary conditions upon which this extension can be made to rest are no longer of the simple type described above, but require for formulation and treatment the introduction of a novel kind of functional, the terminal. A sketch of a theory of terminals was the subject of [8], together with applications to the problem at hand. Here we shall offer a slightly different approach which has the advantage of being more direct, especially where linear equations are concerned.

The main result concerning solutions of analytic elliptic equations in two variables is the fact that they can be "reflected" on analytic boundary conditions and that a local construction can be given to effect this extension. Again the case of linear equations and linear boundary conditions is privileged: apart from conditions about the domain of regularity of the coefficients it can be asserted that for simply connected domains $D$ of definition the solutions of the general equation

$$
u_{x x}+u_{y y}+a(x, y) u_{x}+b(x, y) u_{y}+c(x, y) u+e(x, y)=0
$$

with general linear boundary condition of first order are coextensive with harmonic functions defined in $D$ and satisfying on the same boundary arc the condition $u=0$.

This paper consists of 2 parts. The first part deals exclusively with linear boundary conditions. The second part gives an introduction to a theory of "terminal" operators and applies it subsequently to the reflection of solutions of linear equations on non-linear boundary conditions.

\section{PART I. LiNEAR PRoblems}

1. Extension of harmonic functions across linear boundary conditions. We denote by $D$ a simply connected domain of the $x, y$-plane whose boundary contains a segment $\sigma$ of the $x$-axis with the origin as interior point and such that $D$ contains the portion $y<0$ of a neighborhood of each point of $\sigma$. We express this condition by saying: $D$ is adjacent to $\sigma$, on the side $y<0$. Let $u(x, y)$ be harmonic in $D$ and let $v(x, y)$ be a conjugate harmonic of $u$. Put

$$
z=x+i y, \quad F(z)=u+i v .
$$

Theorem 1. Assume $u$ and $v$ to be conjugate harmonic in $D$, and in $C^{1}$ on $D \cup \sigma$, and to satisfy on $\sigma$ the linear boundary condition

$$
a_{1}(x) u_{x}(x, 0)-a_{2} u_{y}+b_{1} u+b_{2} v=f(x)
$$


where the coefficients $a_{1}(z), a_{2}(z), b_{1}(z), b_{2}(z), f(z)$ are regular in $D \cup \sigma$ and real on $\sigma$ (i.e. for $z=x$ ) and where in $D \cup \sigma$

$$
a_{1}(z)+i a_{2}(z) \neq 0 .
$$

Then $F(z)$ can be continued analytically across $\sigma$ into the mirror image $\bar{D}$ of $D$.

Proof. As in [7] we consider the differential equation in $D$

$$
\begin{aligned}
a_{1}(z)\left(F^{\prime}(z)+G^{\prime}(z)\right) & +i a_{2}(z)\left(-F^{\prime}(z)+G^{\prime}(z)\right) \\
& +b_{1}(z)(F(z)+G(z))+i b_{2}(-F+G)=2 f(z) .
\end{aligned}
$$

In view of (1.2) it can be solved with respect to $G^{\prime}(z)$. We prescribe the initial condition $G(0)=\bar{F}(0)=u-i v(0,0)$ and express the solution $G(z)$ of (1.3) by a well-known explicit formula $\phi$ in terms of the coefficients and $F(z)$ and $F^{\prime}(z)$. On account of our hypotheses about the coefficients and $F, G(z)$ is regular in $D$, and continuous on $D \cup \sigma$. The simple connectivity of $D$ results in the integrals entering $\phi$ being independent of the path which joins the origin to $z$. On $\sigma$, the formula $\phi$ gives the unique solution of (1.3) for the specialization $z=x$ and initial value $G(0)=\bar{F}(0)$.

Now consider in $\bar{D}$ the function $F_{1}(z)$ defined by

$$
F_{1}(z)=\bar{G}(\bar{z}) \text {. }
$$

Since $\bar{z}$ lies in $D, F_{1}$ is defined in $\bar{D}$ and regular there. Furthermore $F_{1}(z)$ assumes on $\sigma$ continuous boundary values. I say that on $\sigma$ we have $F_{1}(x)=F(x)$. This is true for $x=0$, since we let $\bar{F}(0)=G(0)$. If we substitute in (1.3) $z=x$ and $G(x)=\bar{F}(x)$, we satisfy (1.3), for upon this substitution (1.3) reduces to (1.1). Now the uniqueness of the solution of (1.3) for $z=x$ implies that $G(x)$ be identical with $\bar{F}(x)$. Hence $F(z)$ is regular in $D$, continuous in $D \cup \sigma, F_{1}(z)$ is regular in $\bar{D}$, continuous in $\bar{D} \cup \sigma$, and on $\sigma, F$ and $F_{1}$ agree. Thus according to a classical theorem, $F_{1}(z)$ is the analytic extension of $F$ into $\bar{D} \cup \sigma$. The theorem is proved.

REMARK. If we replace in (1.1) the derivative $u_{y}$ by $-v_{x}$ and perform an integration with respect to $x$, we free the boundary condition of any reference to derivatives of $u$ and $v$, obtaining a condition $\left(1.1^{\prime}\right)$. Now if we knew of $u$ and $v$ only their continuity in $D \cup \sigma$, together with (1.1') on $\sigma$ and harmonic conjugation in $D$, we could repeat the above reasoning as follows: While formula $\phi$ contained the derivative $F^{\prime}(z)$, we can remove the reference to $F^{\prime}(z)$ by an integration by parts and obtain a new formula $\phi_{1}$ which expresses $G(z)$ in terms of the coefficients and their derivatives and of $F$ and integrals 
of such. Now the proof proceeds as before, since the uniqueness of the solution of an ordinary differential equation is established by considering only the integrated form of the equation. Under circumstances such as arise frequently in the calculus of variations, it may be easier to ascertain boundary conditions of type (1.1') rather than the hypotheses of the theorem as stated above.

2. Example of a harmonic function of three variables. We show in this section that the analog of Theorem 1 for harmonic functions of three variables is incorrect. Let $x, y, z$ be three real variables and consider the auxiliary function

$$
U(x, y, z)=\int_{-\infty}^{z} e^{-z+t}\left(x^{2}+y^{2}+t^{2}\right)^{-1 / 2} d t
$$

defined for all $x, y, z$ with the exception of the points of the positive $z$-axis. Upon approach to a point of the positive $z$-axis $U$ tends to $\infty$ and therefore the derivative of $U$ in a fixed direction other than the $z$-direction cannot remain bounded upon approach to the positive $z$ axis.

$U$ is harmonic. In fact, consider an integral of form $\int_{-\infty}^{z} e^{-z+t} f(x, y, t) d t$ where $f$ has continuous second derivatives which remain bounded at infinity. We have

$$
\begin{aligned}
\frac{\partial}{\partial z} \int_{-\infty}^{z} e^{-z+t} f(x, y, t) d t & =-\int_{-\infty}^{z} e^{-z+t} f(x, y, t) d t+f(x, y, z) \\
& =\int_{-\infty}^{z} e^{-z+t} \frac{\partial}{\partial t} f(x, y, t) d t
\end{aligned}
$$

and similarly

$$
\frac{\partial^{2}}{\partial z^{2}} \int_{-\infty}^{z} e^{-z+t} f(x, y, t) d t=\int_{-\infty}^{z} e^{-z+t} \frac{\partial^{2}}{\partial t^{2}} f(x, y, t) d t .
$$

Now Laplace's equation follows for $U$ since it holds for $f(x, y, z)$ $=\left(x^{2}+y^{2}+z^{2}\right)^{1 / 2}$. Moreover, by (2.1),

$$
U_{z}+U=\left(x^{2}+y^{2}+z^{2}\right)^{-1 / 2} .
$$

Take an arbitrary plane $p$ through the origin which does not contain the $z$-axis. Denote by $\partial / \partial n$ that linear combination with constant coefficients of the first partial derivatives which is the derivative in the direction normal to $p$. Let $D$ be the halfspace bounded by $p$ which contains the negative $z$-axis and let $\bar{D}$ be its mirror image with respect to $p$. Put 


$$
u(x, y, z)=(\partial / \partial n) U(x, y, z) .
$$

Then (2.2) yields $u_{z}+u=0$ on $p$. Furthermore, $u$ is harmonic in $D$ and continuous with all derivatives in $D \cup p$ minus the origin $O$. Evidently $u$ can be continued as harmonic function across $p$ minus $O$, but does not stay bounded upon approach to the positive $z$-axis, hence is singular there. The plane $p$ can be chosen so as to make an arbitrarily small angle with the $z$-axis. Therefore $u$ becomes an example of a harmonic function on a half-space adjacent to a plane $p$, continuous up to and including $p$ minus $O$, and which satisfies on $p$ minus $O$ a linear homogeneous boundary condition of first order with constant coefficients. Yet $u$ cannot be continued across $p$ as harmonic function into a domain which contains a line through $O$ of some fixed but arbitrarily small angle with $p$.

This example illustrates the difference between the behavior of harmonic functions of two variables where extension into the full mirror image domain of the domain of existence was proved, and that of harmonic functions of three variables where even under the most restrictive assumptions as to the nature of the boundary condition, the assumed domain of existence and its boundary, the analogous conclusions may be erroneous.

3. Linear boundary conditions for linear elliptic equations. We return to the notations of $\$ 1$ concerning $D$ and $\sigma$. We suppose that $u(x, y)$ be real and continuous with its first derivatives in $D \cup \sigma$ and satisfy in $D$ the differential equation

$$
u_{z \bar{z}}+(A(z, \bar{z})+i B(z, \bar{z})) u_{z}+(A-i B) u_{\bar{z}}+C(z, \bar{z}) u=0
$$

where the symbols are defined by

$$
\begin{aligned}
& z=x+i y, \quad \bar{z}=x-i y, \\
& \partial / \partial z=1 / 2(\partial / \partial x-i \partial / \partial y), \\
& \partial / \partial \bar{z}=1 / 2(\partial / \partial x+i \partial / \partial y),
\end{aligned}
$$

and where we assume that $A(z, \zeta), B(z, \zeta), C(z, \zeta)$ are analytic functions of $z$ and $\zeta$ for $z$ in $D \cup \sigma \cup \bar{D}, \zeta$ in $D \cup \sigma \cup \bar{D}$ and furthermore that

$$
\bar{A}(z, \zeta)=A(\bar{\zeta}, \bar{z}), \bar{B}(z, \zeta)=B(\bar{\zeta}, \bar{z}), \bar{C}(z, \zeta)=C(\bar{\zeta}, \bar{z})
$$

Regarding (3.3) we remark that every function of $x$ and $y$ which is real and analytic in $x$ and $y$ near the origin becomes, upon introduction of $z$ and $\bar{z}$ instead of $x$ and $y$, the specialization for $\zeta=\bar{z}$ of a function $A(z, \zeta)$ analytic in $z$ and $\zeta$ and which satisfies (3.3). This is true for real constants, for the function $x$ which is the specialization of 
$(z+\zeta) / 2$ and for $y$ which is the specialization of $-i(z-\zeta) / 2$ for $\zeta=\bar{z}$; it furthermore is true for all sums and products of these functions and their limits. It thus becomes evident that (3.1) is the form of the general linear homogeneous elliptic equation with real analytic coefficients whose terms of highest order coincide with the Laplacian. The assumptions about the coefficients made above are more special only in that they require analyticity in all of the stated domain. Incidentally, the restriction that $u(x, y)$ be real and the corresponding restriction (3.3) of the coefficients plays no essential role in the following considerations and was made only in order to link the subject of our investigation with the customary notion of second order equations. Another remark concerns the supposed homogeneity of (3.1). It would have been possible to treat the nonhomogeneous equation by the same method, but we have chosen to restrict the generality for the sake of shorter formulae and brevity of expression.

Let us write, for real $x$ and $y$,

$$
u(x, y)=U(z, \bar{z}) .
$$

Our first aim is to construct an analytic function $U(z, \zeta)$ whose arguments $z$ and $\zeta$ range independently over $D \cup \sigma \cup \bar{D}$, and which for $\zeta=\bar{z}$ reduces to (3.4).

Although in later sections of this work we attempt to dispense with the explicit expressions of those functionals which furnish extensions such as the one presently to be performed, here in the linear case these expressions are simple enough and connected with the familiar notion of Riemann's function, so that they can serve as an example of, and introduction to, the more abstract notions which the complexity of the nonlinear case requires in their stead.

Denote by $M[v]$ the expression

$$
M[v(z, \zeta)]=v_{z \zeta}-(a v)_{z}-(b v)_{\zeta}+c v
$$

where

$$
\begin{aligned}
& a(z, \zeta)=A(z, \zeta)+i B(z, \zeta) \\
& b(z, \zeta)=A(z, \zeta)-i B(z, \zeta) \\
& c(z, \zeta)=C(z, \zeta)
\end{aligned}
$$

The Riemann function $R\left(z^{0}, \zeta^{0} ; z, \zeta\right)$ is a function of four complex arguments, each ranging independently over $D \cup \sigma \cup \bar{D}$, and satisfying in $z, \zeta$ the equation

$$
M[R]=0 .
$$

It fulfills the further conditions 


$$
\begin{array}{r}
R\left(z^{0}, \zeta^{0} ; z^{0}, \zeta^{0}\right)=1, \\
R_{z}\left(z^{0}, \zeta^{0} ; z, \zeta^{0}\right)-b\left(z, \zeta^{0}\right) R\left(z^{0}, \zeta^{0} ; z, \zeta^{0}\right)=0, \\
R_{\zeta}\left(z^{0}, \zeta^{0} ; z^{0}, \zeta\right)-a\left(z^{0}, \zeta\right) R\left(z^{0}, \zeta^{0} ; z^{0}, \zeta\right)=0,
\end{array}
$$

or their integrated form

$$
\begin{aligned}
& R\left(z^{0}, \zeta^{0} ; z, \zeta^{0}\right)=\exp \int_{z^{0}}^{z} b\left(t, \zeta^{0}\right) d t \\
& R\left(z^{0}, \zeta^{0} ; z^{0}, \zeta\right)=\exp \int_{\zeta^{0}}^{\zeta} a\left(z^{0}, t\right) d t .
\end{aligned}
$$

The construction of Riemann's function for complex arguments proceeds in a manner entirely analogous to the case of real arguments: identical formulae furnish the successive approximations, only the intervening integrals must be interpreted as Cauchy integrals of complex functions rather than as real integrals of real functions. To make matters specific, let us join the point $z^{0}$ to $z$ by a path $(p)$ of $D \cup \sigma \cup \bar{D}$, and $\zeta^{0}$ to $\zeta$ by a path $(\pi)$ of $D \cup \sigma \cup \bar{D}$. The Cartesian product of $(p)$ and $(\pi)$ yields a two-dimensional surface $S^{2}$ of the fourdimensional space of complex $z$ and $\zeta$. Now (3.8') yields first the value of $R$ for $z$ on $(p)$ and $\zeta=\zeta^{0}$, and for $\zeta$ on $(\pi)$ and $z=z^{0}$. These determinations serve to introduce the first approximation on $S^{2}$ of $R$, denoted by $R^{1}\left(z^{0}, \zeta^{0} ; z, \zeta\right)$ and solution of $R_{z \zeta}^{1}=0$. The successive approximations are then obtained by solving for $k=1,2, \cdots$ the equation

$$
R_{z \zeta}^{k+1}=\left(a(z, \zeta) R^{k}\right)_{z}+\left(b R^{k}\right)_{\zeta}-c R^{k}
$$

with the initial condition $R^{k+1}\left(z^{0}, \zeta^{0} ; z, \zeta^{0}\right)=R^{1}\left(z^{0}, \zeta^{0} ; z, \zeta^{0}\right)$ for $z$ on $(p)$ and $R^{k+1}\left(z^{0}, \zeta^{0} ; z^{0}, \zeta\right)=R^{1}\left(z^{0}, \zeta^{0} ; z^{0}, \zeta\right)$ for $\zeta$ on $(\pi)$. The successive approximations converge uniformly as in the purely real case and furnish $R\left(z^{0}, \zeta^{0} ; z, \zeta\right)$ for $z, \zeta$ on $S^{2}$. Now we note that all the successive approximations depend on $(p)$ and $(\pi)$ through finitely many applications of the following processes: integration in $z$ from $z^{0}$ to $z$ of an analytic function of $z$ and $\zeta$, integration in $\zeta$ of an analytic function of $z$ and $\zeta$, and integration of an analytic function of $z$ and $\zeta$ over the product of $(p)$ and $(\pi)$. Now every one of these processes yields a result which is independent of the choice of $(p)$ and $(\pi)$ and depends only on the endpoints $z$ and $\zeta$. The limit function is continuous in $z, \zeta ; z^{0}, \zeta^{0}$ as each of these four variables ranges over $D \cup \sigma \cup \bar{D}$. Since each of the approximations is analytic in all four variables so is the limit function. Moreover, as in the real case, $R$ satisfies in $z^{0}, \zeta^{0}$ the adjoint equation $L[v]=0$ to be defined presently. 
The proof of this last fact needs indeed no repetition because its truth in the real case implies by analytic continuation its truth in the complex domain indicated.

Let us set

$$
L[]=\partial^{2} / \partial z \partial \zeta+a(z, \zeta) \partial / \partial z+b(z, \zeta) \partial / \partial \zeta+c(z, \zeta)
$$

with the meaning (3.6) of the coefficients. Consider the special $S^{2}$ which is the Cartesian product of a $z$-path $(p)$ of $D \cup \sigma$ and a $\zeta$-path $(\pi)$ where now $(\pi)$ is the conjugate path of $(p)$ and where $(p)$ begins at the origin and ends at $z^{0}$. Accordingly $(\pi)=(\bar{p})$ remains in $\bar{D} \cup \sigma$ and ends at $\zeta^{0}=\bar{z}^{0}$. Take the "diagonal" of $S^{2}$, consisting of those points $z, \zeta$ with $z$ on $(p)$ and $\zeta$ on $(\not)$ for which $z=\xi$, and define for these points $U(z, \zeta)=U(z, \bar{z})$ in agreement with (3.4) as the (real) solution of (3.1), and define, in accordance with (3.2') for points of the diagonal of $S^{2},\left.U_{z}(z, \zeta)\right|_{\zeta=\bar{z}}=U_{z}(z, \bar{z})$ and $\left.U_{\zeta}(z, \zeta)\right|_{\zeta=\bar{z}}=U_{\bar{z}}(z, \bar{z})$. We wish to extend $U$ into $S^{2}$ as function of $z$ and $\zeta$ according to the equation $L[U]=0$. We have, precisely as in the real case, the identity (3.10) $-U M[v]+v L[U] \rrbracket=(U v)_{z \zeta}-\left(U v_{\zeta}\right)_{z}-\left(U v_{z}\right)_{\zeta}+(a U v)_{z}+(b U v)_{5}$.

Integrate (3.10) over the "triangle" of $S^{2}$, bounded by the diagonal $d$, the side of points $z$ on $(p), \zeta=0$ and the side of points $z=z^{0}, \zeta$ on $(\not)$ and substitute for $v$ the function $R\left(z^{0}, 0 ; z, \zeta\right)$. We find for the hypothetical solution $U(z, \zeta)$ on $S^{2}$ the identity

$$
0=\oint(U v)_{z} d z-\oint U\left(v_{z}-b v\right) d z+\oint U\left(v_{\zeta}-a v\right) d \zeta
$$

Hence in view of (3.8)

$$
\begin{aligned}
U\left(z^{0}, 0\right)= & U(0,0) R\left(z^{0}, 0 ; 0,0\right) \\
& +\int_{d}\left[\left(U(z, \bar{z}) R\left(z^{0}, 0 ; z, \bar{z}\right)\right)_{z}-U\left(R_{z}-b R\right)\right] d z \\
& +\int_{d} U(z, \bar{z})\left(R_{\zeta}-a R\right) d \bar{z} .
\end{aligned}
$$

(3.12) is a special case of the more general formula in which $(z, \zeta)$ is a general point of the triangle, and which is obtained by integrating over a subtriangle of corners $(z, \bar{z}),(z, \zeta)$ and $(\bar{\zeta}, \zeta)$ and which is

$$
\begin{aligned}
U(z, \zeta)= & U(\bar{\zeta}, \zeta) R(z, \zeta ; \bar{\zeta}, \zeta)+\int_{d^{\prime}} U(t, \bar{t})\left(R_{\bar{t}}(z, \zeta ; t, \bar{t})-a(t, \bar{t}) R\right) d \bar{t} \\
& +\int_{d^{\prime}}\left[(U(t, \bar{t}) R(z, \zeta ; t, \bar{t}))_{t}-U\left(R_{t}-b R\right)\right] d t
\end{aligned}
$$


where $d^{\prime}$ is the part of the diagonal from $(\bar{\zeta}, \zeta)$ to $(z, \bar{z})$.

In particular we have the special case

$$
\begin{aligned}
U\left(0, \zeta^{0}\right)= & U\left(\bar{\zeta}^{0}, \zeta^{0}\right) R\left(0, \zeta^{0} ; \bar{\zeta}^{0}, \zeta^{0}\right) \\
& +\int_{d^{\prime}} U(t, \bar{t})\left(R_{7}\left(0, \zeta^{0} ; t, \bar{t}\right)-a(t, \bar{t}) R\right) d \bar{t} \\
& +\int_{d^{\prime}}\left[\left(U(t, \bar{t}) R\left(0, \zeta^{0} ; t, \bar{t}\right)\right)_{t}-U(t, \bar{t})\left(R_{t}-b R\right)\right] d t .
\end{aligned}
$$

The apparent asymmetry of (3.12) and (3.12') could easily be removed by integrating out the first term of the second integral, due regard being taken of the meaning of the derivative $\partial / \partial t$ on $d^{\prime}$.

Finally, integration in (3.11) over the quadrilateral $(0,0),(z, 0)$, $(z, \bar{z}),(0, \bar{z})$ yields the expression of $U(z, \bar{z})$ in terms of $U(t, 0)$ and $U(0, \bar{t})$ as

$$
\begin{aligned}
U(z, \bar{z})= & -U(0,0) R(z, \bar{z} ; 0,0)+U(z, 0) R(z, \bar{z} ; z, 0) \\
& +U(0, \bar{z}) R(z, \bar{z} ; 0, \bar{z}) \\
& -\int_{(0,0)}^{(z, 0)} U(t, 0)\left(R_{t}(z, \bar{z} ; t, 0)-b(t, 0) R\right) d t \\
& -\int_{(0,0)}^{(0, \bar{z})} U(0, \bar{t})\left(R_{\bar{z}}(z, \bar{z} ; 0, \bar{t})-a(0, \bar{t}) R\right) d \bar{t},
\end{aligned}
$$

a formula of fundamental importance for the problem of reflection of $U$ on boundary conditions along $\sigma$.

On the left hand of (3.13) we have suppressed the reference to the path $(p)$ which enters the construction in an essential way. Let us prove that the result is independent of $(p)$. We must show that the line integral in (3.13) does not depend on $d^{\prime}$ where $d^{\prime}$ is an arbitrary path in the plane of real $(x, y)$, leading in $D \cup \sigma$ from $(\xi, \zeta)$ to $(z, \bar{z})$. But this is immediate since by (3.1) and (3.7)

$$
\begin{aligned}
(U(t, \bar{t}) R(z, \zeta ; t, \bar{t}))_{t i}-\left(U\left(R_{t}-b R\right)\right)_{i}- & \left(U\left(R_{\bar{t}}-a R\right)\right)_{t} \\
& =-U M[R]+R L[U]=0 .
\end{aligned}
$$

The next fact is the analyticity of $U(z, \zeta)$. We must show $\partial U(z, \zeta) / \partial \bar{z}$ $=0$ and $\partial U(z, \zeta) / \partial \bar{\zeta}=0$. Now the first of these is immediate since $R$ depends analytically on $z$ and the contribution of the integrals in (3.13) amounts, upon differentiation with respect to $\bar{z}$, to

$$
\left.U(z, \bar{z})\left(R_{\bar{t}}(z, \zeta ; t, \bar{t})-a(t, \bar{t}) R\right)\right|_{t=z, \bar{z}=\bar{z}}
$$

and vanishes by (3.8). The equation $\partial U(z, \zeta) / \partial \bar{\zeta}=0$ follows similarly if we first replace 


$$
U(\bar{\zeta}, \zeta) R(z, \zeta ; \bar{\zeta}, \zeta)+\int_{d^{\prime}}(U(t, \bar{t}) R(z, \zeta ; t, \bar{t}))_{t} d t
$$

by

$$
U(z, \bar{z}) R(z, \zeta ; z, \bar{z})-\int_{d^{\prime}}(U(t, \bar{t}) R(z, \zeta ; t, \bar{t}) z d \bar{t} .
$$

The analyticity of $U(z, \zeta)$ is of course a classical result due to Picard. Even the above mentioned method of proving it is essentially about 30 years old $[3 ; 5]$. But what matters here is the extent of the domain into which $U(z, \zeta)$ is analytically extensible, in its dependence on the original domain of definition of the solution $u(x, y)$ of (3.1): namely, for $z$ in $D$, and $\zeta$ in $\bar{D}$.

We are now in a position to discuss the simplest linear boundary condition for $u(x, y)=U(z, \bar{z})$ on $\sigma$, which is

$$
u(x, 0)=U(x, x)=\delta(x)
$$

where we suppose that $\delta(z)$ is regular for $z$ in $D \cup \sigma \cup \bar{D}$. We replace in (3.14) $U(z, 0)$ by $f(z)$ and $U(0, z)$ by $g(z)$ and obtain for $z$ on $\sigma$

$$
\delta(z)=-\delta(0) R(z, z ; 0,0)+f(z) R(z, z ; z, 0)+g(z) R(z, z ; 0, z)
$$

$$
\begin{aligned}
& -\int_{0}^{z} f(t)\left(R_{t}(z, z ; t, 0)-b(t, 0) R\right) d t \\
& -\int_{0}^{z} g(t)\left(R_{t}(z, z ; 0, t)-a(0, t) R\right) d t .
\end{aligned}
$$

Notice that in view of $\left(3.8^{\prime}\right) R(z, z ; z, 0)$ and $R(z, z ; 0, z)$ do not vanish for $z$ in $D \cup \sigma \cup \bar{D}$. Observe that $f(z)$ is known through (3.12) for $z$ in $D \cup \sigma$, in terms of the given solution $u(x, y)$, and so is $g(z)$ for $z$ in $\bar{D} \cup \sigma$, through $\left(3.12^{\prime}\right)$. The idea of the analytic continuation of $u(x, y)$ across $\sigma$ is first to extend $f(z)$ and likewise $g(z)$ into $D \cup \sigma \cup \bar{D}$, by using (3.16), which is originally valid only on $\sigma$, throughout $D \cup \sigma \cup \bar{D}$ for the construction of these extensions.

Notice that (3.16) is a Volterra integral equation for $g(z), z$ in $D \cup \sigma$, since $f(z)$ and $\delta(z)$ are known in $D \cup \sigma$. The solution $g(z)$ must therefore exist and be unique in all of $D \cup \sigma$, and be regular in $D$ since the kernel and the terms which do not involve $g(z)$ satisfy the requisite analyticity conditions. Now $g(z)$ is known beforehand to be regular in $\bar{D}$ and continuous in $\bar{D} \cup \sigma$. The above construction of $g(z)$ furnishes therefore the analytic continuation of $g(z)$ into $D \cup \sigma \cup \bar{D}$. In analogous manner the analytic continuation of $f(z)$ into $D \cup \sigma \cup \bar{D}$ 
is effected by interpreting (3.16) as a Volterra integral equation for $f(z)$ in $\bar{D} \cup \sigma$ where $g(z)$ and $\delta(z)$ are known beforehand.

We now utilize (3.14) and obtain $U(z, \bar{z})$ for arbitrary $z$ of $D \cup \sigma \cup \bar{D}$ as analytic extension of $U(z, \bar{z})$ for $z$ in $D \cup \sigma$ as given originally. Hence the

TheOREM 3.1. Reflection of the $C^{1}$-solutions $u(x, y)$ of (3.1) on the boundary condition (3.15) on $\sigma$ is coextensive with the reflection of harmonic functions on the boundary condition $u=0$.

Notice that $U(z, \zeta)$ is known now through (3.13) in all of the Cartesian products of $z$ and $\zeta$ ranging through $D \cup \sigma \cup \bar{D}$.

Our next problem is the general first order boundary condition on $\sigma$

$$
\alpha(x) U_{z}(x, x)+\beta(x) U_{\bar{z}}(x, x)+\gamma(x) U(x, x)+\delta(x)=0
$$

where $\alpha(z), \beta(z), \gamma(z), \delta(z)$ are regular throughout $D \cup \sigma \cup \bar{D}$. This is a real boundary condition if $\bar{\alpha}(\bar{z})=\beta(z)$. We require

$$
\alpha(z) \neq 0, \quad \beta(z) \neq 0 \text { throughout } D \cup \sigma \cup \bar{D} .
$$

Differentiate (3.14) with respect to $z$ and thereafter set $z=\bar{z}$ for $z$ on $\sigma$. We do not write down the lengthy result of this operation, but can content ourselves with ascertaining its structure in terms of $U(z, 0), U(0, z)$ and $U_{z}(z, 0)$.

For this purpose we remark that a function $f(z)$ as well as the Volterra integral $\int_{0}^{z} K(z, t) f(t) d t$ are at the same time, but for an additional term dependent only on $f(0)$, Volterra integrals of $f^{\prime}(t)$ since

$$
\begin{aligned}
\int_{0}^{z} K(z, t) f(t) d t & =f(0) \int_{0}^{z} K(z, t) d t+\int_{0}^{z} K\left(z, t^{\prime}\right)\left(\int_{0}^{t^{\prime}} f^{\prime}(t) d t\right) d t^{\prime} \\
& =f(0) K_{1}(z, 0)+\int_{0}^{z} K_{1}(z, t) f^{\prime}(t) d t \\
K_{1}(z, t) & =\int_{t}^{z} K\left(z, t^{\prime}\right) d t^{\prime} .
\end{aligned}
$$

Accordingly we can write the result of the above differentiation

$$
\begin{aligned}
U_{z}(z, \bar{z})=R(z, \bar{z} ; z, 0) U_{z}(z, 0) & + \text { Volterra integral of } U_{t}(t, 0) d t \\
& + \text { Volterra integral of } U_{z}(0, \bar{t}) d \bar{t} \\
& + \text { known function times } U(0,0), \\
U_{\bar{z}}(z, \bar{z})=R(z, \bar{z} ; 0, \bar{z}) U_{\bar{z}}(0, z) & + \text { terms similar to above. }
\end{aligned}
$$


On setting $z=\bar{z}=x$ for points of $\sigma,(3.17)$ becomes, with $U(z, 0)=f(z)$, $U(0, z)=g(z)$,

$$
\begin{aligned}
\alpha(z) R & (z, z ; z, 0) f^{\prime}(z)+\beta(z) R(z, z ; 0, z) g^{\prime}(z) \\
& \quad+\text { Volterra integral of } f^{\prime}(t) d t+\text { Volterra integral of } g^{\prime}(t) d t \\
= & \text { given analytic function of } z \text { times } U(0,0) \\
& + \text { given analytic function of } z .
\end{aligned}
$$

Again we notice that the coefficients of $f^{\prime}(z)$ and of $g^{\prime}(z)$ do not vanish throughout $D \cup \sigma \cup \bar{D}$ on account of (3.18) and $\left(3.8^{\prime}\right)$. The kernels of the Volterra integrals are analytic functions of their arguments as long as these remain in $D \cup \sigma \cup \bar{D}$. We can therefore solve (3.20) for the function $g^{\prime}(z)$ in $D \cup \sigma$, where $f^{\prime}(z)$ is known and continuous, and determine $g^{\prime}(z)$ as continuous in $D \cup \sigma$ and regular in $D$. Thus $g^{\prime}(z)$ has been extended analytically into $D \cup \sigma \cup \bar{D}$. In a like manner $f^{\prime}(z)$ is analytically extended into the same domain. Again (3.14) yields $U(z, \bar{z})$ for $z$ in $D \cup \sigma \cup \bar{D}$ as analytic function of $z$ and $\bar{z}$, and (3.13) yields $U(z, \zeta)$ as $z$ and $\zeta$ range independently over $D \cup \sigma \cup \bar{D}$. Thus

Theorem 3.2. Let $u(x, y)$ be in $C^{\prime}$ for $z$ in $D \cup_{\sigma}$ and satisfy (3.1) in D. Assume that on $\sigma$ the boundary condition (3.17) is satisfied and that the coefficients $A(z, \zeta), B(z, \zeta), C(z, \zeta), \alpha(z), \beta(z), \gamma(z), \delta(z)$ of equation and boundary condition are regular for $z$ and $\zeta$ in $D \cup \sigma \cup \bar{D}$ and that (3.18) holds. Then $u(x, y)$ can be extended as analytic function into $D \cup \sigma \cup \bar{D}$.

REMARK. The assumptions concerning $C^{\prime}$-continuity of $u$ in Theorem 3.1 could be considerably relaxed. We omit details.

4. A theorem on Cauchy data admitting a solution for $\Delta u=0$ and for $\Delta u+\lambda u=0$. An interesting consequence may be drawn from (3.14) when applied simultaneously to two different differential equations whose solutions share Cauchy data on $\sigma$. We shall restrict the consideration to the equation $U_{z \bar{z}}=0$ (Laplace's equation) and $U_{z \bar{z}}$ $=-\lambda U$, with $\lambda(z, \bar{z}) \neq 0$ and analytic near the origin. In the first case the Riemann function is identically equal to 1 , in the second case $R$ satisfies, by $\left(3.8^{\prime}\right)$

$$
R(z, \bar{z} ; z, 0)=R(z, \bar{z} ; 0, \bar{z})=1
$$

whence

$$
\left.R_{\bar{z}}(z, \bar{z} ; 0, \bar{t})\right|_{z=\bar{z}}+\left.R_{\bar{l}}(z, \bar{z} ; 0, \bar{t})\right|_{z=\bar{z}}=0
$$

and

$$
\left[R_{z} t+R_{z \bar{z}}(z, \bar{z} ; 0, \bar{t})\right]_{\bar{z}=\bar{z}}=0 .
$$


Now $R_{z \bar{z}}+\lambda(z, \bar{z}) R=0, R$ being a solution of the same equation relative to its first two as well as last two arguments. Hence

$$
\left.R_{z \bar{t}}(z, \bar{z} ; 0, \bar{t})\right|_{\bar{l}=\bar{z}}=\lambda(z, \bar{z}) R(z, \bar{z} ; 0, \bar{z}) \neq 0
$$

for $|z|$ sufficiently small.

Differentiation of (3.14) yields under the present circumstances

$$
\begin{aligned}
U_{z}(z, \bar{z})= & -U(0,0) R_{z}(z, \bar{z} ; 0,0)+f^{\prime}(z)-\left.f(z) R_{t}(z, \bar{z} ; t, 0)\right|_{t=z} \\
& -\int_{0}^{z} R_{z t}(z, \bar{z} ; t, 0) f(t) d t-\int_{0}^{\bar{z}} R_{z \bar{z}}(z, \bar{z} ; 0, \bar{t}) g(\bar{t}) d \bar{t}, \\
U(t, 0)= & f(t), \quad U(0, t)=g(t) .
\end{aligned}
$$

We recall that $f(z)$ is known in $D \cup \sigma, g(z)$ in $\bar{D} \cup \sigma$, provided $u(x, y)$ is given in $D \cup_{\sigma}$. Now let $v(x, y)=V(z, \bar{z})$ be harmonic in $D \cup_{\sigma}$ and have the same first derivatives on $\sigma$ as $U$. (3.14) for $V$ is

$$
V(z, \bar{z})=-V(0,0)+F(z)+G(\bar{z})
$$

with $F$ and $G$ having analogous meaning as $f$ and $g$ and with same domain of definition and of regularity. On $\sigma$ we find accordingly

$$
\begin{aligned}
F^{\prime}(z)= & -U(0,0) R_{z}(z, z ; 0,0)+f^{\prime}(z)-\int_{0}^{z} R_{t z}(z, z ; t, 0) f(t) d t \\
& -f(z) R_{t}(z, z ; t, 0)_{t=z}-\int_{0}^{z} R_{t z}(z, z ; 0, t) g(t) d t .
\end{aligned}
$$

Now this equation can be used to extend $g(z)$ across $\sigma$ because the kernel of this Volterra equation for $g(t)$ does not vanish, at least in the neighborhood of the origin. A similar computation will show that $f(z)$ can be extended analytically across $\sigma$ near the origin. Therefore (3.14) yields the analyticity of the Cauchy data themselves. Hence the Theorem (see [6]).

Theorem 4.1. If $v(x, y)$ and $u(x, y)$ are in $C^{\prime}$ in $D \cup \sigma, v(x, y)$ is harmonic in $D$ and $\Delta u+4 \lambda u=0$ in $D$ with $\lambda(x, y) \neq 0$ near the origin and analytic in $x, y$ in a full neighborhood of the origin, and if on $\sigma$ we have $u_{x}=v_{x}, u_{v}=v_{y}$, then $u$ and $v$ can be analytically extended across $\sigma$ near the origin. Hence the Cauchy data are analytical near the origin.

The interest of Theorem 4.1 lies in the following observation. Suppose we call a function of one real variable strictly nonanalytic in an interval if it is analytic in no subinterval. What can be said about the existence of solutions of elliptic equations in a domain $D$ for strictly nonanalytic Cauchy data on $\sigma$ ? Theorem 4.1 asserts that the 
equations $\Delta u=0$ and $\Delta u+4 \lambda u=0$ can never have solutions in $D$ near the origin, for the same set of strictly nonanalytic Cauchy data.

5. Explicit formulae of reflection. In $\$ 3$ the reflection of a solution of (3.1) on boundary conditions on $\sigma$ was based on an extension of the solution into a four-dimensional domain of complex $x$ and $y$ which contains the given domain of definition of $u$. Can the result of this reflection be expressed analytically without reference to any complex extension, as linear functional of the given solution and its first derivatives in its domain of original definition? The answer is in the affirmative and is provided with the aid of certain functions, akin to Riemann's function, which depend on 4 real variables $x, y, X, Y$, satisfy in each pair $x, y$ or $X, Y$ certain differential equations closely connected with (3.1), and which for $x=X, y=-Y$ satisfy a further system of equations embodying the given boundary condition on which the reflection is effected. Although it would be possible to base the existence of these auxiliary functions on the proofs given we shall not pursue this explicit representation here for the sake of brevity.

\section{PaRT II. Nonlinear BOUNDARY CONDITIONS}

6. Terminals. Definitions and elementary consequences. If a solution of the linear equation (3.1) is subject to a nonlinear boundary condition of analytic character what can be asserted relative to the analytic continuation of the solution across this boundary condition? In the case of Laplace's equation it was seen in [7] that the problem of analytic continuation can be solved with the aid of the solution of an ordinary differential equation in the complex plane; the method explained in $\S 1$ of this paper yields the possibility of analytic continuation in this case. It is easily seen by examples that in general no more than a local extension is possible, even for solutions of Laplace's equation.

If Laplace's equation is replaced by the general equation (3.1) the device of solving an ordinary differential equation in order to construct the analytic continuation, is no longer available. We saw in $\$ 3$ how in the case of a linear boundary condition the analytic continuation is reduced to the solution of certain Volterra type integral equations. In the nonlinear case even this is no longer a sufficiently large frame of operations. Our present task is to develop a short theory of functionals adequate for this purpose. It is simpler to do this without reference to the problem under consideration and the following two sections do not require any reference to the theory of partial differential equations. 
We consider a simply connected domain $D$ of the complex $z$-plane with rectifiable boundary $B$. Suppose the origin $O$ to lie in $D \cup B$ and designate by $(p)$ a rectifiable path in $D \cup B$ leading from $O$ to $z$, by $\left(p^{\prime}\right)$ a portion of $(p)$ leading from $O$ to the point $z^{\prime}$ of $(p)$. Denote by $\phi(z)$ a (complex-valued) function of $z$ which is continuous in $D \cup B$. We emphasize that $\phi(z)$ should be admissible whether regular or not. We consider a functional

$$
\Omega(\phi(t) \mid(p), z)
$$

of the function $\phi(t)$ on $(p)$ of endpoint $z$, whose value is a complex number. In practice it may also be necessary to impose an upper limitation on the length of the path $(p)$ and/or the modulus of $\phi$ in $D \cup B$.

$\Omega(\phi(t) \mid(p), z)$ is called a terminal if, whenever $\phi(z)$ is analytic in $D$, the value

$$
\psi=\Omega(\phi \mid(p), z)=\psi(z)
$$

is independent of the path $(p)$ used to connect 0 with $z$, whence for such $\phi, \psi$ becomes a function of the endpoint alone.

A terminal $\Omega(\phi \mid(p), z)$ is said to be continuous if in the above notation for $(p)$ and $\left(p^{\prime}\right)$

$$
\lim _{z^{\prime} \rightarrow z} \Omega\left(\phi \mid\left(p^{\prime}\right), z^{\prime}\right)=\Omega(\phi \mid(p), z) .
$$

A terminal is called regular if $\psi(z)$ is regular in $D$ whenever $\phi(z)$ is regular in $D$.

EXAMPLES. Every function of $\phi(z)$ is a terminal. Sums, products and limits of terminals are terminals. A function of a terminal is a terminal, in particular the reciprocal of a nonvanishing terminal. $\int_{(p)} \phi(t) d t$ is a regular continuous terminal. Notice that here the simple connectivity of $D$ is essential.

Let $\Omega_{\nu}(\phi \mid(p), z), \nu=1,2, \cdots$ be a sequence of regular terminals uniformly bounded for all admissible $\phi, z,(p), \nu$, and which converges as $\nu \rightarrow \infty$. Then the limit is also a regular terminal.

If $\Omega$ is a regular continuous terminal, the transform $\psi(z)$ $=\Omega(\phi \mid(p), z)$ of a function $\phi$ regular in $D$ and continuous in $D \cup B$ is again (regular in $D$ and) continuous in $D \cup B$.

A terminal of a regular continuous terminal, $\Lambda(\Omega)$, is a terminal. This is correct if properly interpreted. Forming $\Lambda$ for a path $(p)$ we need the values of $\psi=\Omega\left(\phi \mid(q), z^{\prime}\right)$ at all points $z^{\prime}$ of $(p)$ which in turn appear as endpoints of certain paths $(q)$ used in forming $\Omega\left(\phi \mid(q), z^{\prime}\right)$. We now require that $(q)=\left(p^{\prime}\right)$ as defined above. Then $\Lambda$ becomes a functional of $\phi$ on $(p)$ alone, and the argument function $\psi$ of $\Lambda(\psi \mid(p), z)$ 
is continuous on $(p)$ by hypothesis about $\Omega$. Furthermore, if $\phi$ is regular in $D, \psi$ is regular in $D$, and since $\Lambda$ is a terminal the value of $\Lambda(\psi \mid(p), z)$ does then not depend on the path $(p)$, only on its endpoint $z$. If $\Lambda$ is a regular and continuous terminal of $\psi, \Lambda(\Omega)$ is a regular continuous terminal of $\phi$.

A trivial extension of the notions occurs when the argument function $\phi$ of $\Omega$ is replaced by a vector function of $n$ components and when the result $\psi$ of the operation $\Omega$ is again a vector of $m$ components.

7. An inversion theorem. We designate by $K(z), \phi(z), \phi(z), \cdots$ vectors of $n$ (complex-valued) components, by $M, M_{1}>M, N, \sigma_{0}$ positive constants, by $\omega(s)$ an increasing function of $s$ with $\omega(0)=0$. We assume $K(z)$ to be continuous in $D \cup B$ and denote by $|K(z)|$ a norm for $K(z)$, e.g. the maximum of the moduli of the components at $z$. Let $s(p)$ stand for the length of $(p)$.

Suppose $\Omega(\phi \mid(p), z)$ to be a regular continuous terminal with $n$ components, defined for all vector functions $\phi$ which are continuous in $D \cup B$ and whose norm does not exceed $M_{1}$, and for all paths $(p)$ with $s(p) \leqq \sigma_{0}$.

Assume the following inequalities for $z$ in $D \cup B, s(p) \leqq \sigma_{0}$ :

$$
\begin{aligned}
& |K(z)|<M \\
& |\Omega(\phi \mid(p), z)| \leqq \omega(s(p)) \\
& |\Omega(\phi \mid(p), z)-\Omega(\tilde{\phi} \mid(p), z)| \\
& \quad \leqq N s(p)|\phi(z)-\tilde{\phi}(z)|+N \int_{(p)}|\phi(t)-\tilde{\phi}(t)||d t| .
\end{aligned}
$$

THEOREM 7.1. Under the stated assumptions there exists a positive $\sigma \leqq \sigma_{0}$ depending only on $M_{1}, M, N, \omega, \sigma_{0}$ such that the equation

$$
\phi(z)=K(z)+\Omega(\phi \mid(p), z)
$$

has one and only one solution $\phi(z)$ existing on all paths $(p)$ of length $s(p) \leqq \sigma$. Moreover, $\phi-K$ is itself a regular continuous terminal $\Omega_{1}$ of $K(z)$ and satisfies inequalities similar to (7.2) and (7.3), but with other constants.

Proof. The construction of the solution $\phi(z)$ of (7.4) is achieved by successive approximations and does not differ in essence from the construction of the solution of an ordinary differential equation in the complex plane.

Set $\phi^{1}(z)=K(z), \phi^{\nu+1}\left(z^{\prime}\right)=K\left(z^{\prime}\right)+\Omega\left(\phi^{\nu} \mid\left(p^{\prime}\right), z^{\prime}\right)$. Put

$$
\Delta^{v}\left(p^{\prime}\right)=\max _{\left(p^{\prime}\right)}\left|\phi^{\nu+1}(t)-\phi^{\nu}(t)\right|
$$


and determine $\sigma>0$ such that

$$
2 N \sigma<1, \quad \sigma \leqq \sigma_{0}, \quad \omega(\sigma)(1-2 N \sigma)^{-1} \leqq M_{1}-M .
$$

Then $\Delta^{1}\left(p^{\prime}\right) \leqq \omega(\sigma) \leqq M_{1}-M$, hence $\Omega\left(\phi^{2} \mid\left(p^{\prime}\right), z^{\prime}\right)$ is defined. Thus

$$
\begin{aligned}
& \Delta^{2}\left(p^{\prime}\right) \leqq 2 N \sigma \Delta^{1}\left(p^{\prime}\right) \leqq \omega(\sigma) 2 N \sigma, \\
& \left|\phi^{3}\right| \leqq\left|\phi^{1}\right|+\Delta^{1}+\Delta^{2} \leqq M+\omega(\sigma)(1+2 N \sigma) \leqq M_{1}, \\
& \Omega\left(\phi^{3} \mid\left(p^{\prime}\right), z^{\prime}\right) \text { is defined for } s\left(p^{\prime}\right) \leqq \sigma, \text { etc. }
\end{aligned}
$$

The sequence $\phi^{\nu}$ converges uniformly and all $\left|\phi^{\nu}\right|<M_{1}$.

Now $\phi^{1}(z)=K(z)$ is a regular continuous terminal of $K(z)$. Assume that $\phi^{\nu}$ is a regular continuous terminal of $K(z)$. Since $\Omega\left(\phi^{\nu}\right)$ is a regular continuous terminal of $\phi^{\nu}$ it becomes a regular continuous terminal of $K(z)$. Now the uniform convergence of $\phi^{v}$ guarantees that the limit $\phi$ of $\phi^{\nu}$ as $\nu \rightarrow \infty$ is a solution of (7.4), by (7.3). It follows that $\phi-K$ is a regular continuous terminal $\Omega_{1}$ of $K$. We have by (7.2), (7.3)

$$
\begin{gathered}
\Omega_{1}(K \mid(p), z)=\Omega(\phi \mid(p), z) ;\left|\Omega_{1}(K \mid(p), z)\right| \leqq \omega(s(p)), \\
\left|\Omega_{1}(K \mid(p), z)-\Omega_{1}(\tilde{K} \mid(p), z)\right|=\mid \Omega(\phi|(p, z)-\Omega(\tilde{\phi} \mid(p), z)| \\
\leqq N|\phi(z)-\tilde{\phi}(z)| s(p)+N \int_{(p)}|\phi(t)-\tilde{\phi}(t)| d t, \\
|K(z)-\tilde{K}(z)|=|\phi(z)-\tilde{\phi}(z)-(\Omega(\phi \mid(p), z)-\Omega(\tilde{\phi} \mid(p), z))| \\
\geqq|\phi(z)-\tilde{\phi}(z)|(1-N s(p))-N \int_{(p)}|\phi-\tilde{\phi}||d t|, \\
\int_{(p)}|K(t)-\tilde{K}(t)||d t| \\
\geqq \int_{(p)}|\phi-\tilde{\phi}||d t|\left(1-2 N \sigma_{1}\right), \text { for } s(p)<\sigma_{1} \leqq \sigma ; \\
|K(z)-\tilde{K}(z)| s(p)+\int_{(p)}|K(t)-\tilde{K}(t)| \mid d t \\
\geqq|\phi(z)-\tilde{\phi}(z)|\left(1-N \sigma_{1}\right) s(p)+\int_{(p)}|\phi(t)-\tilde{\phi}(t)||d t|\left(1-3 N \sigma_{1}\right) \\
\geqq\left(1-3 N \sigma_{1}\right)\left[|\phi(z)-\tilde{\phi}(z)| s(p)+\int_{(p)}|\phi(t)-\tilde{\phi}(t)||d t|\right] .
\end{gathered}
$$

Hence a relation analogous to (7.3) holds for $\Omega_{1}(K \mid(p), z)$ with $N$ replaced by $N /\left(1-3 N \sigma_{1}\right)^{-1}$ provided $0<\sigma_{1} \leqq \sigma, 3 N \sigma_{1}<1$. Finally, (7.4) has no other solution than the one constructed. For otherwise 
let $z^{\prime}$ be that point on $(p)$ for which the norm of the difference of two solutions $\phi, \tilde{\phi}$ is maximum, say $=\mu$. Then by (7.4) and (7.3)

$$
\mu \leqq 2 N \sigma \mu \text {, or } \mu=0 \text { since } 2 N \sigma<1 \text {. }
$$

A corollary of Theorem 7.1 which has the appearance of greater generality relates to the equation

$$
\phi(z)=K(z)+\Omega(\phi, K \mid(p), z)
$$

where now $\Omega$ is allowed to depend on $K$ as well. To see this we only have to introduce a vector of $2 n$ components $\psi$ whose first $n$ components are those of $\phi$, whose last $n$ components those of $K$. The $n$-vector $K$ on the right of (7.6) is to be replaced by a $2 n$-vector whose first $n$ as well as last $n$ components are those of $K$. The terminal $\Omega(\phi, K \mid(p), z)$ thus becomes the first $n$ components of a terminal $\Omega^{*}(\psi \mid(p), z)$ whose last $n$ components are identically zero.

THEOREM 7.2. The conclusions of Theorem 7.1 hold good for (7.6) instead of (7.4) provided the terminal $\Omega(\phi, K \mid(p), z)$ considered as terminal $\Omega^{*}$ of the $2 n$-vector $\psi=(\phi, K)$ satisfies the conditions of Theorem 7.1 relative to $\psi$.

8. Typical reflection problem. Let $\Omega(\phi \mid(p), z)$ be a regular continuous terminal of $\phi$ for $z$ in a circle $\gamma$ about the origin, defined for all $\phi$ which are continuous in $\gamma$ and whose norm remains there inferior to $M_{1}$, and for all paths $(p)$ with $s(p) \leqq \sigma$. Assume that in $\gamma$ the inequalities (7.2) and (7.3) hold.

Denote by $\beta$ the segment of the $x$-axis contained in $\gamma$, by $D$ and $\bar{D}$ the two adjacent semicircles of $\gamma$. Let $\phi^{\circ}(z)$ be defined in $D \cup \beta$, regular in $D$ and continuous on $D \cup \beta$, and with $\left|\phi^{0}\right|<M_{1}$. Let $K^{0}(z)$ be defined in $\bar{D} \cup \beta$, regular in $\bar{D}$ and continuous in $\bar{D} \cup \beta$, and with $\left|K^{0}(z)\right|<M, M<M_{1}$.

Now suppose that on $\beta$ there holds

$$
\phi^{0}(x)=K^{0}(x)+\Omega\left(\phi^{0} \mid x\right)
$$

where it is understood that the path leading from $O$ to $x$ is the segment $O x$.

Then $\phi^{\circ}(z)$ can be analytically continued across $\beta$ near $O$.

Proof. The inversion Theorem (7.1) yields a unique solution in $\bar{D} \cup \beta$ for sufficiently short paths $s(p)$, of

$$
\phi^{0}(z)=K^{0}(z)+\Omega\left(\phi^{0} \mid(p), z\right) .
$$

This $\phi^{0}(z)$ is regular in $\bar{D}$ near the origin and coincides with $\phi^{0}(x)$ on $\beta$. being the unique solution of (8.1). Thus $\phi^{0}(z), z$ in $D \cup \beta$, has been extended across $\beta$ near $O$ as analytic function. 
9. Nonlinear boundary condition for linear differential equations. We shall now show how the reflection of the solution of (3.1) on a nonlinear boundary condition of first order can be reduced to the situation dealt with in the preceding section.

THEOREM 9.1. Let $u(x, y)=U(z, \bar{z})$ be a solution of (3.1) in the part $y<0$ of a circle $\gamma$ about the origin of the $x, y$-plane, continuous with first derivatives in $y \leqq 0$. Assume that $u(x, 0)$ satisfies on $y=0$ a real relation

$$
u_{y}=h\left(x, u, u_{x}\right)
$$

where $h$ is an analytic function of its arguments near $x=0, u=u(0,0)$, $u_{x}=u_{x}(0,0)$. Then $u(x, y)$ can be continued analytically across $y=0$ near the origin.

Proof. (9.1) assumes in terms of $U, U_{z}, U_{\bar{z}}$ the following two forms

$$
\begin{aligned}
& U_{\bar{z}}=H_{1}\left(z, U, U_{z}\right), \\
& U_{z}=H_{2}\left(z, U, U_{\bar{z}}\right),
\end{aligned}
$$

where the arguments of $U$ and its derivatives are $x, x$ and $z=x$. For (9.1) becomes

$$
U_{\bar{z}}=U_{z}+i h\left(x, U, U_{z}+U_{\bar{z}}\right)
$$

which can be solved algebraically with respect to $U_{z}$ since $1-i \partial h / \partial u_{x}$ $\neq 0$, giving (9.2). Similarly for (9.3). Note that $H_{1}$ and $H_{2}$ are power series of $z, U-U(0,0), U_{z}-U_{z}(0,0)$ and of $z, U-U(0,0), U_{z}$ $-U_{\tilde{z}}(0,0)$ respectively.

Denote by $D$ the part $y<0$ of $\gamma$, by $\bar{D}$ its mirror image on $\beta$, the diameter of $D$. Our construction consists of three steps the first of which is the repetition of a construction of $\$ 3$ and yields the formula (3.12) for $U(z, 0), z$ in $D \cup \beta$, and (3.12') for $U(0, \zeta), \zeta$ in $\bar{D} \cup \beta$. Note that $U(z, 0)$ is regular for $z$ in $D$ and in $C^{1}$ for $z$ in $D \cup \beta$, while $U(0, \zeta)$ is regular for $\zeta$ in $\bar{D}$ and in $C^{1}$ for $\zeta$ in $\bar{D} \cup \beta$. In terms of $U(z, 0)$ and $U(0, \bar{z})$ we have the expression (3.14) for $U(z, \bar{z}), z$ in $D \cup \beta$.

Set

so that

$$
\begin{aligned}
& U_{z}(z, 0)-U_{z}(0,0)=F(z), \\
& U_{\zeta}(0, \zeta)-U_{\zeta}(0,0)=G(\zeta),
\end{aligned}
$$

$$
\begin{aligned}
& U(z, 0)=U(0,0)+z U_{z}(0,0)+\int_{0}^{z} F(t) d t, \\
& U(0, z)=U(0,0)+z U_{\zeta}(0,0)+\int_{0}^{z} G(t) d t .
\end{aligned}
$$


Substitute into (3.14) and find

$$
\begin{aligned}
U(z, \bar{z})= & \alpha_{1}(z, \bar{z})+R(z, \bar{z} ; z, 0) \int_{0}^{z} F(t) d t+R(z, \bar{z} ; 0, z) \int_{0}^{\bar{z}} G(t) d t \\
& +\int_{0}^{z} V_{1}(z, \bar{z} ; t) F(t) d t+\int_{0}^{\bar{z}} V_{2}(z, \bar{z} ; \bar{t}) G(\bar{t}) d \bar{t}
\end{aligned}
$$

where $\alpha_{1}(z, \bar{z}), V_{1}(z, \bar{z} ; t), V_{2}(z, \bar{z} ; \bar{t})$ are certain analytic functions of $z, \bar{z}, t, \bar{t}$ when $z$ and $t$ are in $\gamma$, and $\alpha_{1}(0,0)=U(0,0)$. Differentiation of (9.4) with respect to $z$, followed by replacing $\bar{z}$ by $z$ for $z$ on $\beta$, yields on $\beta$

$$
\begin{aligned}
U_{z}(z, z)= & \rho_{1}(z) F(z)+\int_{0}^{z} V_{3}(z, t) F(t) d t \\
& +\int_{0}^{z} V_{4}(z, t) G(t) d t+\alpha_{1}(z)
\end{aligned}
$$

where $V_{3}(z, t), V_{4}(z, t), \alpha_{1}(z)$ are certain analytic functions of $z$ and $t$ in $\gamma$, and $\alpha_{1}(0)=U_{z}(0,0), \rho_{1}(z)=R(z, z ; z, 0)$. Similarly differentiation of (9.4) with respect to $z$ followed by replacing $\bar{z}$ by $z$ for $z$ on $\beta$, yields on $\beta$

$$
\begin{aligned}
U_{\bar{z}}(z, z)= & \rho_{2}(z) G(z)+\int_{0}^{z} V_{5}(z, t) F(t) d t \\
& +\int_{0}^{z} V_{6}(z, t) G(t) d t+\alpha_{2}(z)
\end{aligned}
$$

where $V_{5}(z, t), V_{6}(z, t), \alpha_{2}(z)$ are analytic for $z$ and $t$ in $\gamma$ and $\alpha_{2}(0)$ $=U_{\bar{z}}(0,0), \rho_{2}(z)=R(z, z ; 0, z)$. We recall that by $\left(3.8^{\prime}\right) \rho_{1}(z)$ and $\rho_{2}(z)$ do not vanish.

We now take from (9.4) the expression of $U(z, z)-U(0,0)$, from (9.5) that of $U_{z}(z, z)-U_{z}(0,0)$ and from (9.6) that of $U(z, z)-U_{z}(0,0)$ and substitute these in (9.3). The result is a formula

$$
F(z)=\Omega(F \mid(p), z)+K(z)
$$

with $K(z)=\left(\alpha_{1}(z)-U_{z}(0,0) / \rho_{1}(z)\right.$ and $\Omega$ a certain functional the nature of which we must describe. First of all $\Omega$ depends also on $G(z)$. We have suppressed in (9.7) the mention of $G(z)$ because we wish to utilize (9.7) in $\bar{D} \cup \beta$ and we know that $G(z)$ is regular in $\bar{D}$ and continuous in $\bar{D} \cup \beta$. Note that $F(0)=G(0)=0$ and that $F(z)$ enters $\Omega$ only through Volterra integrals $\int_{0}^{z} V(z, t) F(t) d t$ with analytic $V(z, t)$ so that $\Omega$ is independent of $(p)$ if $F(z)$ is regular in $\bar{D}$ and continuous 
in $\bar{D} \cup \beta$. Thus $\Omega$ is seen to be a continuous terminal. It is regular since in $\bar{D}$ the result of substituting a regular $F(z)$ makes $\Omega$ regular in $\bar{D}$. Finally, there is some concentric subcircle $\gamma^{\prime}$ of $\gamma$ and some positive constant $M_{1}$ such that for $|F(z)| \leqq M_{1}, z$ in $\gamma^{\prime} \cap(\bar{D} \cup \beta)$, the power series $H_{2}$ of $U-U(0,0), U_{\bar{z}}-U_{\bar{z}}(0,0), z$ will converge and represent an analytic function, provided $s(p)$ is inferior to some positive $\sigma_{0}$. Moreover, in view of the continuity of $G(z)$ in $\bar{D} \cup \beta$ an inequality of form (7.2) is readily established. Now, as to the Lipschitz condition (7.3), we notice that $\left(U_{z}(z, z)-U_{z}(0,0)\right) / \rho_{1}(z)$, considered through (9.5) as a functional of $F(z)$, obviously satisfies a Lipschitz condition of form (7.3), and so does $\left(U_{\bar{z}}(z, z)-U_{\bar{z}}(0,0)\right)$ from $(9.6)$ and $U(z, z)$ $-U(0,0)$ from (9.4). Substitution in the power series $H_{2}$ thus makes $H_{2}$ subject to a Lipschitz condition (7.3). If the circle $\gamma^{\prime}$ has sufficiently small radius we are sure that $K(z)=\left(\alpha_{1}(z)-\alpha_{1}(0)\right) / \rho_{1}(z)$ remains in $\gamma^{\prime} \cap(\bar{D} \cup \beta)$ absolutely inferior to a constant $M$, chosen positive and $<M_{1}$.

Having thus established for the terminal $\Omega$ of (9.7) the conditions of the inversion Theorem 7.1, we proceed to solve (9.7) for $F(z)$ in $(|z|<\sigma) \cap(\bar{D} \cup \beta)$ where $\sigma$ is the constant of Theorem 7.1. The solution is unique and continuous, and is regular in $(|z|<\sigma) \cap \bar{D}$. But on $\beta$ (9.7) expresses the boundary condition which the given $u(x, y)$ satisfies by hypothesis. Hence on $\beta,(9.7)$ holds for $F(x)$ as just constructed as well as for $F(x)$ as given by (3.12); recall the definition $\left(^{*}\right)$ of $F(z)$ in terms of $U(z, 0)$. The two determinations are equal on account of uniqueness. Therefore we have obtained an analytic extension of $F(z)$ from its original domain $D$ of definition into $((\bar{D} \cup \beta)$ $\cap(|z|<\sigma)) \cup D ; F(z)$ has been continued analytically across $\beta$ near the origin.

In analogous manner (9.2) is used to establish the analytic continuation of $G(z)$ across $\beta$ near $O$ from its original domain of definition $\bar{D}$. This completes step 2 of the construction.

The final step of the construction is this. We know that (3.14) holds for $z$ in $D \cup \beta$. But the functions $U(z, 0)$ and $U(0, \zeta)$, being respectively the integrals of $F(z)$ and $G(\zeta)$, are now known in a full neighborhood of the origin. Hence (3.14) yields $U(z, \bar{z})$ as an analytic function in a full neighborhood of the origin. This is the statement of Theorem 9.1.

In the following bibliography only the case of two independent variables is recorded. The first to prove analytic extensibility for general linear equations and special linear boundary conditions was Hadamard [3] who based the extension on the Cauchy-Kowalewski Theorem and Green's formula employing the fundamental solution. 
The precise domain of extension (Theorems 3.1 and $3.1^{\prime}$ ) is new. A special case of Theorem 9.1 was treated in [1].

\section{BIBLIOGRAPHY}

1. P. R. Garabedian, H. Lewy, M. Schiffer, Axially symmetric cavitational flow, Ann. of Math. vol. 56 no. 3 (1952) pp. 560-602.

2. Robert Gerber, Sur une condition de prolongement analytiques des fonctions harmoniques, C. R. Acad. Sci. Paris vol. 233 (1951) pp. 1560-1562.

3. Jacques Hadamard, Le problème de Cauchy et les équations aux dérivées partielles linéaires hyperboliques, Appendix III, French Edition, Paris, 1932, Hermann, p. 512 ff.

4. —_ Mémoire sur le problème d'analyse relatif a l'équilibre des plaques élastiques encastrées. Mémoires de divers savants présentés à l'Acad. Sci. vol. 32, ser. 2, no. 4 (1908) pp. 1-128.

5. Hans Lewy, Eindeutigkeit der Lösung des Anfangsproblems einer elliptischen Differentialgleichung zweiter Ordnung in zwei Veränderlichen, Math. Ann. vol. 104 (1931) pp. 325-339.

6. - Sur une nouvelle formule dans les equations linéaires elliptiques et une application au problème de Cauchy, C. R. Acad. Sci. Paris (1933), pp. 112-113.

7. - A note on harmonic functions and a hydrodynamical application, Proc. Amer. Math. Soc. vol. 3 (1952) pp. 111-113.

8. - $-A$ theory of terminals and the reflection laws of partial differential equations, Technical Report No. 4 (ONR) Stanford, 1952.

University of California, Berkeley 